\title{
Composition Chimique Et Activité Antimicrobienne Des Huiles Essentielles De Deux Espèces De Lavande : Lavandula Dentata Spp. Dentata Et Lavandula Peduncultata Spp. Pedunculata
}

\section{Lamia Bachiri}

Equipe de Microbiologie du Sol et de l'Environnement, Université My

Ismail Faculté des Sciences, B.P : 11201, Meknès (Maroc)

Département de Protection des Plantes et de l'Environnement, Ecole

Nationale d'Agriculture, B.P : S/40 - Meknès (Maroc)

\section{Mohamed Bammou}

Equipe de Microbiologie du Sol et de l'Environnement, Université My Ismail Faculté des Sciences, B.P : 11201, Meknès (Maroc)

Equipe de l'Environnement et Santé, Département de Biologie, Université My Ismail, Faculté des Sciences et Techniques, B.P 509, Errachidia (Maroc)

\section{Ghizlane Echchegadda}

Département de Protection des Plantes et de l'Environnement, Ecole

Nationale d'Agriculture, B.P : S/40 - Meknès (Maroc)

\section{Jamal Ibijbijen}

Equipe de Microbiologie du Sol et de l'Environnement, Université My Ismail Faculté des Sciences, B.P : 11201, Meknès (Maroc)

\section{Lhoussaine El Rhaffari}

Equipe de Valorisation des Plantes Médicinales et Aromatiques de la Région Meknès-Tafilalt, Université My Ismail, Faculté des Sciences, B.P : 11201, Meknès (Maroc)

\section{Zoubida Haloui}

Equipe de Valorisation des Plantes Médicinales et Aromatiques de la Région Meknès-Tafilalt, Université My Ismail, Faculté des Sciences, B.P : 11201,

Meknès (Maroc)

\section{Laila Nassiri}

Equipe de Microbiologie du Sol et de l'Environnement, Université My Ismail Faculté des Sciences, B.P : 11201, Meknès (Maroc) 


\section{Abstract}

The aim of the present work is to compare the chemical composition, antibacterial and antifungal activity of two spontaneous species of lavender "Lavandula stoechas L." and "Lavandula dentata L." in Morocco. A phytochemical screening was performed on the aerial part of invested plants. The qualitative variation was revealed in the secondary metabolites of the species studied. Subsequently, the dosage of the crude organic extracts showed that the polyphenol content was higher in the methanol fraction, with an important yield in L. dentata. Also, the essential oils of the aerial part, obtained by hydrodistillation gave higher yields in L. dentata than $L$. pedunculata. Finally, tests of the antibacterial and antifungal activity of the essential oils of the two species were performed on four strains of bacterial pathogens (Staphylococcus aureus "Gram+," and Escherichia coli; Klebsiella pneumoniae and Proteus mirabilis which are Gram-), and three phytopathogenic fungi : Rhizopus stolonifer, Aspergillus brasiliensis and Penicillium expansum, The results obtained showed that the essential oil of L. dentata showed a strong antibacterial activity against the studied strains, particularly against $S$. aureus. While the antifungal activity revealed that $L$. pedunculata has a remarkable antifungal power against the fungi tested in comparison with L. dentata.

Keywords: Lavandula dentata, Lavandula pedunculata, phytochemistry, essential oil, antibacterial activity, antifungal activity

\section{Résumé}

Le présent travail porte sur la comparaison de la composition chimique et l'activité antibactérienne et antifongique de deux espèces de lavande, spontanées au Maroc "Lavandula dentata spp. dentata et Lavandula peduncultata spp. pedunculata." Un criblage phytochimique a été réalisé sur la partie aérienne des plantes investies ; la variation qualitative a été révélée au niveau des métabolites secondaires des espèces étudiées. Par la suite, le dosage des extraits organiques bruts a montré que la teneur en polyphénols est plus élevée au niveau de la fraction méthanolique, avec un rendement important chez la lavande dentée. De leur côté, les huiles essentielles de la partie aérienne, obtenues par hydrodistillation ont donné des rendements plus importants chez L. dentata par rapport à L. pedunculata. Enfin les tests de l'activité antibactérienne et antifongique des huiles essentielles des deux espèces ont été réalisés sur quatre souches bactériennes pathogènes : Staphylococcus aureus «Gram+», Escherichia coli, Klebsiella pneumoniae et Proteus mirabilis «Gram»»et sur trois champignons phytopathogènes: Rhizopus stolonifer, Aspergillus brasiliensis et Penicillium expansum. Les résultats obtenus ont montré que l'huile 
essentielle de $L$. dentata a montré une forte activité antibactérienne contre les souches étudiées, notamment contre $S$. aureus. Par contre l'activité antifongique a révélé que $L$. pedunculata avait un pouvoir antifongique très remarquable vis-à-vis des champignons testés en comparaison avec $L$. dentata.

Mots-clés : Lavandula dentata- L.pedunculata-Phytochimie- Huile essentielle- Activité antibactérienne- Activité antifongique

\section{Introduction}

La flore marocaine est un réservoir important de plantes aromatiques et médicinales dont les principes actifs peuvent être utilisés en cosmétique, aromathérapie, pharmacologie, parfumerie, et en industrie agroalimentaire (Bellakhdar, 1997).

Dans les sociétés modernes, celle marocaine entre autres, on note une forte tendance vers les produits naturels, tant pour les soins de santé, de beauté ou comme additifs alimentaires en tant qu'arômes, conservateurs ou qu'épices et condiments.

Outre le recours aux divers types d'extraits de plantes dont les huiles essentielles pour éviter le risque d'effets secondaires des médicaments ou le développement d'une résistance à de tels remèdes de la part des microbes, l'utilisation de ces substances naturelles comme biopesticides pourrait aider à atténuer l'impact négatif des intrants chimiques sur les réseaux trophiques, la santé humaine, et l'environnement global.

Les huiles essentielles de plusieurs espèces ont ainsi fait l'objet de nombreuses études qui ont révélé leur multifonctionnalité et l'ampleur de leurs perspectives d'application dans divers domaines en rapport avec la variabilité chimique de leurs constituants (Hyldgaard et al., 2012). Il s'agit aussi bien d'activités anticancéreuses, antivirales, anti-acariennes, antioxydantes, qu'antifongiques et antimicrobiennes sur un large spectre de microorganismes (Burt, 2004 ; Bakkali et al., 2008 ; Lang et al., 2011).

Dans ce cadre, les lavandes, grâce à leurs vertus aromatiques, médicinales, sont parmi les plantes populaires au Maroc. Diverses investigations ethnobotaniques sont en faveur de leur utilisation en thérapie, cosmétique, odoriférantes de linge en plus de leur valeur pastorale (Bachiri et al., 2015). Aussi, sur la base de ces données ethnobotaniques, des recherches scientifiques s'imposent-elles dans le but de valoriser le patrimoine ethnopharmacologique relatif à cette ressource végétale et de préserver la santé humaine via ses métabolites secondaires, source potentielle d'agents antimicrobiens. Déjà, il a été rapporté que les huiles essentielles des lavandes ont des propriétés antispasmodiques et sédatives (Cavanagh al., 2002) ainsi que des activités acaricides (Perrucci et al., 1996); antibactériennes 
(Dadalioglu et al., 2004 ;Moon et al., 2006), antifongiques (Angioni et al., 2006 ; Zuzarte et al., 2009) et anti-oxydantes (Matos et al., 2009 ; Benabdelkader et al., 2011 ; Benabdelkader, 2012).

Par ailleurs, une autre voie à explorer chez les lavandes est l'éventualité d'une variabilité interspécifique et infraspécifique ; en effet, l'activité biologique des huiles essentielles dépend de leur composition chimique qui, elle, est déterminée par plusieurs facteurs, intrinsèques : le génotype, et extrinsèques : le biotope, les conditions de récolte des plantes, les méthodes d'extraction...

Aussi, l'objectif de la présente étude est la comparaison de deux espèces de lavande autochtone au Maroc, L. dentata spp. dentata et L. peduncultata spp. Pedunculata quant à leur composition chimique et l'activité de leurs huiles essentielles vis-à-vis de quatre souches bactériennes pathogènes humaines (Staphylococcus aureus, Escherichia coli, klebsiella

pneumoniae et Proteus mirabilis et trois souches fongiques phytopathogènes (Aspergillus brasiliensis, Penicillium expansum et Rhizopus stolonifer).

Les résultats permettraient de déduire la relation entre le profil chimique et la taxonomie d'une part, la taxonomie et l'activité antimicrobienne d'autre part.

\section{Matériel et Méthodes}

\section{Matériel végétal}

Les échantillons de la partie aérienne (tiges, feuilles et fleurs) de Lavandula pedunculata (communément appelée Lhlhal) et ceux de Lavandula dentata (Lkhzama marrakchia) ont été récoltés en Mai 2015, respectivement aux environs d'Azrou, dans le Moyen Atlas central et d'Oujda, dans les monts de l'oriental marocain.

\section{Microorganismes étudiés}

Les souches utilisées pour le test antibactérien sont : Staphylococcus aureus (Gram+) et Escherichia coli, Klebsiella pneumoniae et Proteus mirabilis (Gram-), toutes d'origine clinique ;

- Staphylococcus aureus: Le staphylocoque doré (Staphylococcus aureus) est la souche de staphylocoque la plus fréquemment rencontrée en pathologie humaine et vétérinaire. Elle partage avec la bactérie Escherichia coli le triste privilège d'être au premier rang des germes responsables d'infections nosocomiales (infections contractées à l'hôpital) (Chambers, 1997). S. aureus est également en tête des bactéries responsables d'intoxications alimentaires (Le Loir et Gautier, 2010).

- Escherichia coli: bactérie naturellement présente dans la flore intestinale. Si la plupart des souches de cette bactérie sont sans danger pour 
la santé, certaines sont à l'origine d'infections intestinales plus ou moins graves. Les pathologies généralement associées aux souches $E$. coli pathogènes sont des infections urinaires, des diarrhées, des gastro-entérites, des infections urinaires...

- $\quad$ Klebsiella pneumoniae : entérobactérie, déterminant des infections respiratoires (pneumonies, abcès pulmonaires, pleurésies), des infections intestinales et urinaires. Elle a un effet cytotoxique sur les épithéliums des voies aériennes et peut être responsable de pneumonies nosocomiales .

- $\quad$ Proteus mirabilis : fait partie de la flore intestinale normale chez l'humain, mais susceptible de causer des infections quand elle quitte l'intestin; souvent en cause dans les infections des voies urinaires, qu'elles soient nosocomiales ou non.

Quant aux champignons phytopathogènes testés, il s'agit de Rhizopus stolonifer, Aspergillus brasiliensis et Penicillium expansum fournis par le laboratoire d'Environnement et Santé, de la faculté des sciences et techniques d'Errachidia.

- Rhizopus stolonifer : Espèce saprophyte survivant très facilement sur/ ou dans le sol ou sur les débris végétaux; pénétrant dans les fruits par l'intermédiaire de la moindre blessure, il les envahit avec son mycélium causant des pourritures molles et liquides des fruits (fraises, raisins, abricots, pêches, prunes, melon...) et des légumes (pois, carottes, concombre, courgette, tomates, aubergines...), ainsi que la moisissure noire, observée communément à la surface du pain et d'autres aliments amylacés.

- Aspergillus brasiliensis: champignon filamenteux, de type moisissure ; comme la plupart des Aspergillus, c'est un saprophyte, qui, à la faveur de blessures, pénètre dans certains fruits ou semences de céréales, Allium et cucurbitacées avant et pendant le stockage.

- Penicillium expansum : agent de pourriture des fruits (surtout pommes et poires), il peut contaminer les jus de fruits et compotes. Il peut produire une mycotoxine la patuline «expansine» (responsable de la «pourriture bleue des pommes ») produisant un contaminant commun des Maloideae (pommes, poires, coings) et source de pertes financières et de risque sanitaire importants, en tant que premier contaminant des jus de fruits, compotes, cidres et autres sous-produits de la pomme. Il contamine aussi diverses cultures maraîchères et arboricoles.

\section{Tests phytochimiques}

Afin de mettre en évidence la présence ou l'absence de certains composés chimiques appartenant aux principales familles des métabolites secondaires, des tests phytochimiques spécifiques basés sur des réactions de coloration, turbidité ou de précipitations, utilisant les techniques analytiques décrites par Bruneton (1993), Dohou (2003), Bekro et al, (2007) ont été conduits. 


\section{Extraction des huiles essentielles (HE)}

L'extraction de l'huile essentielle à partir des parties aériennes des lavandes a été effectuée par hydrodistillation dans un appareil de type Clevenger (Clevenger, 1928). Trois distillations ont été réalisées pendant $3 \mathrm{~h}$ par ébullition dans un ballon de $2 \mathrm{~L}$, de $100 \mathrm{~g}$ de matériel végétal frais avec 1 L d'eau. L'huile obtenue fut conservée dans un flacon sombre à une température de $-4{ }^{\circ} \mathrm{C}$, jusqu'à son utilisation.

\section{Préparation des extraits organiques bruts :}

La préparation des extraits organiques bruts a été effectuée par épuisement successif au soxhlet, de $15 \mathrm{~g}$ de poudre végétale, par trois solvants organiques, à polarité croissante $(150 \mathrm{ml}$ de chaque solvant) : Hexane, Acétate d'éthyle et Méthanol. Après évaporation des solvants, les différents extraits secs ont été conservés à $4^{\circ} \mathrm{C}$.

\section{Dosage des polyphénols totaux}

La teneur en polyphénols totaux des extraits des plantes étudiées a été déterminée par la méthode de Vermerius et al., (2006) en utilisant le réactif de Folin-Ciocalteu.

Dans des tubes à essais, un volume de $0,1 \mathrm{ml}$ de chaque extrait a été introduit avec $2 \mathrm{ml}$ d'une solution de carbonate de sodium à $2 \%$; après agitation et incubation pendant 5 minutes, $0.1 \mathrm{ml}$ du réactif Folin- Ciocalteu $(1 \mathrm{~N})$ fut ajouté. Le mélange obtenu a été incubé à la température ambiante et à l'abri de la lumière pendant 30 minutes; l'absorbance a ensuite été mesurée à l'aide d'un spectrophotomètre à $700 \mathrm{~nm}$.

La quantification des polyphénols totaux a été faite à l'aide d'une courbe d'étalonnage réalisée par l'acide gallique à différentes concentrations. Les résultats sont exprimés en mg équivalent d'acide gallique par g d'extrait. Activité antifongique in vitro des huiles essentielles extraites des deux espèces de lavande

\section{-Par la méthode de micro-atmosphère}

La méthode de micro-atmosphère utilisée (Soylu et al., 2010) repose sur l'évaluation à une température d'incubation donnée, de l'activité inhibitrice de la fraction volatile d'une huile essentielle sur la croissance mycélienne. Les boîtes de pétri de $90 \mathrm{~mm}$ de diamètre ont été préparées par addition de $20 \mathrm{ml}$ de milieu PDA en surfusion (20 ml de milieu PDA offre $80 \mathrm{ml}$ d'air dans chaque boite). L'inoculation a été réalisée en surface, sous forme de dépôts de disques mycéliens $(6 \mathrm{~mm})$ au centre de la boite. Des morceaux de papier filtre (wattman $90 \times 20 \mathrm{~mm}$ de diamètre) ont été placés au fond du couvercle de chaque boîte de pétri et imprégnés avec différentes 
concentrations de l'HE : 0 (témoin), 10, 20 et 40 $\mu 1 /$ disque (équivalent à 0 ; 0,$125 ; 0,25$ et $0,5 \mu \mathrm{l} / \mathrm{ml}$ d'air, respectivement).

Immédiatement, les boîtes furent scellées à l'aide de parafilm pour éviter l'évaporation de l'HE, puis incubées à l'obscurité à une température de $25 \pm 2{ }^{\circ} \mathrm{C}$ pendant 3 jours pour Rhizopus stolonifer et 6 jours pour Aspergillus brasiliensis et Penicillium expansum.

Pour chaque espèce fongique et chaque concentration, trois répétitions ont été réalisées et trois boîtes ont été utilisées par essai.

La croissance mycélienne a été suivie en mesurant la moyenne de deux diamètres perpendiculaires passant par le centre de chaque boite. La fongi-toxicité, exprimée en terme de pourcentage d'inhibition de la croissance du mycélium (I\%) a été calculée selon la formule de Pandey et al.(1982) :

$\mathrm{I} \%=\left(\mathrm{D}_{\mathrm{T}}-\mathrm{D}_{\mathrm{H}} / \mathrm{D}_{\mathrm{T}}\right) \times \mathbf{1 0 0}$

Avec : $\mathbf{D}_{\mathbf{T}}$ : le diamètre de la culture du champignon (en $\mathrm{mm}$ ) dans un milieu sans huile (témoin);

DH: le diamètre de la culture du même champignon dans un milieu en présence de l'huile.

\section{Détermination de l'activité antibactérienne par la méthode de diffusion ou de l'aromatogramme}

L'évaluation de l'activité antibactérienne a été réalisée par la méthode de diffusion en gélose décrite par Rhayour (2002).

À partir d'une culture pure de 24 heures sur gélose nutritive, des suspensions de concentration équivalente au $0,5 \mathrm{McFarland}\left(\sim 10^{8} \mathrm{UFC} / \mathrm{ml}\right)$ de chacune des souches bactériennes à tester ont été préparées.

Des disques de papier Wattman de $6 \mathrm{~mm}$ de diamètre, imprégnés par un volume connu de l'huile essentielle $(5,10,15$ et $20 \mu \mathrm{l})$ ont été placés à la surface d'un milieu gélosé (Agar de Muller Hinton « $A M H »)$; un disque imprégné d'eau distillée stérile servant de témoin négatif a été également déposé sur la surface de la gélose inoculée. De la même façon, un témoin positif a été assuré par l'utilisation d'antibiotiques appropriés.

Les boîtes de Pétri ont été incubées à $37^{\circ} \mathrm{C}$ pendant 24 heures; la lecture des résultats a été faite par la mesure du diamètre de la zone d'inhibition en $\mathrm{mm}$.

\section{Analyse statistique}

L'évaluation de l'effet des huiles essentielles testées sur la croissance des champignons et des bactéries a été investie par une analyse de la variance (ANOVA) ; puis, les moyennes et les écarts types ont été calculés à l'aide du logiciel Graph Pad et le test de Tukey au seuil de probabilité 5\% fut utilisé pour la comparaison des moyennes. 


\section{Résultats et Discussion}

\section{Rendement en huiles essentielles}

Les rendements moyens en huiles essentielles ont été calculés en fonction de la matière végétale sèche de la partie aérienne de la plante. Les échantillons de $\boldsymbol{L}$. dentata ont fourni un taux d'environ $2,60 \% \pm 0,01$; qui est plus élevé que celui obtenu avec $\boldsymbol{L}$. peduncultata, de 1,83\% $\% 0,07$.

Cette différence du rendement en huiles essentielles entre les deux espèces de lavande est normale, puisque le rendement dépend de plusieurs facteurs à savoir l'espèce, le génotype, en plus de l'environnement, la période de récolte, le lieu de séchage et l'origine géographique et (Svoboda et al., 1982 ;Smallfield, 2001).

\section{Screening phytochimique}

Dans le tableau 1 sont présentés les résultats du screening phytochimique mené dans le but de rechercher, dans les extraits de $L$. Pedunculata et $L$. dentata, les différentes classes de substances secondaires, à savoir : les flavonoïdes, les saponines, les tanins, les alcaloïdes, les anthocyanes, les terpènes et les composés réducteurs (Tableau 1). Ces résultats sont classés en fonction des différents critères d'observation :

$++++\quad$ : Réaction très positive; +++ : Réaction positive; ++ : Réaction moyennement positive ; + : Réaction douteuse ; - : Test négatif

Tableau 1 : Résultats du screening phytochimique des deux espèces de lavande étudiée

\begin{tabular}{|c|c|c|c|c|}
\hline \multicolumn{3}{|c|}{ Métabolites secondaires } & L.dentata & L.peduncultata \\
\hline \multicolumn{3}{|c|}{ Alcaloïdes } & - & - \\
\hline \multicolumn{3}{|c|}{ Catéchiques } & ++ & +++ \\
\hline \multicolumn{3}{|c|}{ Galliques } & ++++ & +++ \\
\hline \multicolumn{3}{|c|}{ Anthocyanes } & ++++ & ++++ \\
\hline \multicolumn{3}{|c|}{ Flavones } & ++++ & +++ \\
\hline \multicolumn{3}{|c|}{ Flavanones } & - & - \\
\hline Flavonoïdes & \multicolumn{2}{|c|}{ Flavanonols } & - & - \\
\hline & \multicolumn{2}{|c|}{ Leucoanthocyanes } & - & - \\
\hline & \multicolumn{2}{|c|}{ Catéchols } & +++ & +++ \\
\hline \multirow{3}{*}{$\begin{array}{c}\text { Dérivés } \\
\text { anthracéniques }\end{array}$} & \multicolumn{2}{|c|}{ Anthracéniques libres } & - & - \\
\hline & \multirow[t]{2}{*}{$\begin{array}{l}\text { Anthracéniques } \\
\text { combinés }\end{array}$} & $\begin{array}{l}\text { O- } \\
\text { hétérosides }\end{array}$ & - & +++ \\
\hline & & $\begin{array}{c}\text { C- } \\
\text { hétérosides }\end{array}$ & ++++ & ++++ \\
\hline \multirow{2}{*}{$\begin{array}{l}\text { Composés } \\
\text { réducteurs }\end{array}$} & \multicolumn{2}{|c|}{ Oses et holosides } & - & ++ \\
\hline & \multicolumn{2}{|c|}{ Mucilages } & - & - \\
\hline \multirow[b]{2}{*}{ Terpénoïdes } & \multicolumn{2}{|c|}{ Stérols et tri terpènes } & ++++ & ++++ \\
\hline & \multicolumn{2}{|c|}{ Saponosides } & - & - \\
\hline
\end{tabular}


D'une part, le criblage phytochimique de la partie aérienne des deux lavandes étudiées a mis en évidence la présence de plusieurs composés chimiques réputées avoir des activités biologiques intéressantes (activité antibactérienne, activité antifongique, activité antioxydante...). Il s'agit entre autres des substances polyphénoliques dont les tanins catéchiques et galliques, des flavonoïdes (anthocyanes, flavones et catéchols), des stérols et triterpènes, en plus des anthracéniques combinés (C-hétérosides et $\mathrm{O}$ hétérosides) et des composés réducteurs (Oses et holosides).

Les résultats montrent des différences entre les deux espèces, tant au niveau qualitatif (composés différents) qu'au niveau quantitatif proportions différentes de certains composés) d'autre part. Ainsi, au niveau des tanins galliques et des flavones, une réaction très positive est notée chez la lavande dentée contre une réaction positive chez la lavande pédonculée ; de même, la réaction est moyennement positive avec les tanins catéchiques avec la première espèce et elle est positive avec la seconde. Quant aux Ohétérosides, oses et holosides, absents chez la lavande dentée, ils sont fortement présents chez la lavande pédonculée.

Par conséquent, cette différence chimique qualitative des deux lavandes, peut se répercuter sur leurs activités biologiques. Outre l'effet du biotope (climat et sol), les deux espèces étant issues de deux localités différentes, le génotype peut de son côté, être responsable d'une telle différence.

\section{Dosage des polyphénols totaux}

L'extraction avec des solvants à polarité croissante, permet de séparer les composés d'une plante selon leur degré de solubilité. Un intérêt particulier est dédié aux polyphénols car la plupart des effets pharmacologiques des plantes sont attribués à leur richesse en ces métabolites. Aussi, les taux de polyphénols totaux enregistrés dans les extraits hexaniques, Acétate éthyliques, méthanoliques des espèces de lavande étudiées sont résumés dans la figure 1.

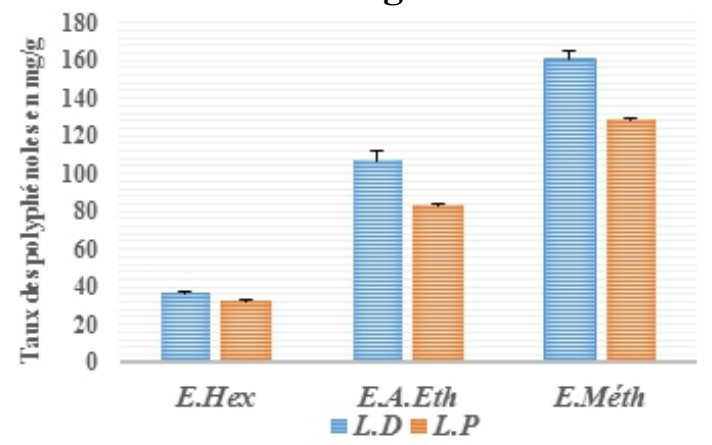

Figure 1 : Taux de polyphénols des extraits hexaniques (E.Hex), Acétate éthylique(E.A.Eth) et méthanoliques (E.Méth) 
Il apparait que le taux des polyphénols varie proportionnellement avec la polarité des solvants d'extraction. En effet, les extraits méthanoliques détiennent les taux les plus élevés (entre 128,45 et 160,24mg/g), suivis par les extraits Acétate-éthyliques (entre 83,01et 106,99 mg/g) alors que les teneurs les plus faibles sont enregistrées avec les extraits hexaniques, les valeurs étant comprises entre 32,26 et $36,90 \mathrm{mg} / \mathrm{g}$. Ces résultats concordent avec ceux d'autres travaux qui ont affirmé que le méthanol est le solvant approprié pour une forte récupération des polyphénols (Falleh et al., 2008) et (Barchan et al., 2015).

Par ailleurs, quel que soit le type de solvant, la comparaison entre les deux espèces (figure 1) laisse voir que la lavande dentée présente des teneurs en polyphénols plus élevées que celles de la lavande pédonculée et l'écart est plus marqué au niveau des extraits méthanoliques et acétate-éthyliques.

\section{Activité antibactérienne et antifongique Activité antibactérienne}

Les résultats de l'activité antimicrobienne reportés dans le Tableau 2 montrent que les huiles essentielles des deux espèces de Lavande étudiées sont actives vis-à-vis des bactéries Gram (-) (E. coli, K. pneumoniae et $P$. mirabilis) et Gram (+) (S. aureus). Sauf quelques exceptions (mentionnées en surbrillance dans le tableau 2), l'effet inhibiteur des HE testées augmente significativement avec le volume.

Aussi, les résultats indiquent que l'activité antibactérienne de l'huile essentielle de la lavande pédonculée s'avère être moins active que celle de la lavande dentée ; ceci apparait de façon très nette vis à vis de $S$. aureus et $K$. pneumoniae

Tableau 2 : Diamètres des zones d'inhibition ( $\mathrm{mm}$ ) illustrant l'activité antibactérienne des huiles essentielles issues des différentes lavandes étudiées.

\begin{tabular}{|c|c|c|c|c|c|}
\hline \multirow[t]{2}{*}{ Espèce } & \multirow{2}{*}{$\begin{array}{c}\text { Volumes } \\
(\mu 1)\end{array}$} & \multicolumn{4}{|c|}{ Zones d'inhibition (mm) } \\
\hline & & S. aureus & E. coli & $\begin{array}{c}K . \\
\text { pneumoniae }\end{array}$ & P. mirabilis \\
\hline L. dentata & $\begin{array}{c}5 \\
10 \\
15 \\
20\end{array}$ & $\begin{array}{c}11,10 \pm 0,10^{\mathrm{a}} \\
13,07 \\
\pm 0,12^{\mathrm{b}} \\
19,03 \pm 0,06^{\mathrm{c}} \\
20,10 \pm 0,10^{\mathrm{d}}\end{array}$ & $\begin{array}{l}10,03 \pm 0,06^{\mathrm{e}} \\
11,10 \pm 0,10^{\mathrm{f}} \\
11,23 \pm 0,21^{\mathrm{f}} \\
12,10 \pm 0,17^{\mathrm{g}}\end{array}$ & $\begin{array}{c}10,10 \pm 0,10^{\mathrm{h}} \\
11,03 \pm 0,06^{\mathrm{i}} \\
12,17 \pm 0,29^{\mathrm{j}} \\
18,03 \pm 0,00^{\mathrm{k}}\end{array}$ & $\begin{array}{c}8,10 \pm 0,10^{1} \\
10,10 \pm 0,10^{\mathrm{m}} \\
10,23 \pm 0,21^{\mathrm{m}} \\
12,30 \pm 0,26^{\mathrm{n}}\end{array}$ \\
\hline $\begin{array}{c}\text { L.p } \\
\text { edunculata }\end{array}$ & $\begin{array}{c}5 \\
10 \\
15 \\
20\end{array}$ & $\begin{array}{l}8,03 \pm 0,06^{\mathrm{A}} \\
11,10 \pm 0,10^{\mathrm{B}} \\
14,10 \pm 0,10^{\mathrm{C}} \\
15,13 \pm 0,15^{\mathrm{D}}\end{array}$ & $\begin{array}{c}10,03 \pm \\
0,0 \\
6^{\mathrm{E}} \\
10,07 \pm 0,12 \\
\mathrm{E} \\
14,10 \pm 0,10^{\mathrm{F}} \\
15,07 \pm 0,12^{\mathrm{G}}\end{array}$ & $\begin{array}{c}8,13 \pm 0,15^{\mathrm{H}} \\
9,03 \pm 0,06^{\mathrm{I}} \\
12,13 \pm 0,15^{\mathrm{J}} \\
13,1 \pm 0,10^{\mathrm{K}}\end{array}$ & $\begin{array}{c}9,00 \pm 0,00^{\mathrm{L}} \\
9,03 \pm 0,06^{\mathrm{L}} \\
9,07 \pm 0,12^{\mathrm{L}} \\
10,10 \pm 0,10^{\mathrm{M}}\end{array}$ \\
\hline
\end{tabular}


ATB

$\mathrm{CN}(\mathrm{S})$

$=19 \pm 0,00$
IMP (S)

$=26 \pm 1,00$
$\operatorname{IMP}(\mathrm{S})$
$=26 \pm 0,00$

$\operatorname{IMP}(\mathrm{S})$

$=26 \pm 1,00$

Pour chaque espèce, les moyennes sur la même colonne suivies de lettres différentes sont significativement différentes selon le test de Tukey au seuil de probabilité de $5 \%$.

$\mathrm{ATB}$ : antibiotique de référence $\mathrm{CN}_{30}$ : Gentamicine $(30 \mu \mathrm{g} / \mathrm{disc})$; $\mathrm{IPM}_{10}$ : Imipenème $(10 \mu \mathrm{g} / \mathrm{disc})$

En outre, en comparaison avec les antibiotiques de référence, si l'effet des HE testées reste modéré pour la majorité des concentrations utilisées, il faut noter que vis-à-vis de S. aureus, à partir du traitement «15 $\mu 1 »$, l'action inhibitrice de l'huile essentielle de Lavandula dentata dépasse l'effet de l'antibiotique de référence ; ces résultats corroborent avec ceux mentionnés par d'autres chercheurs (Imelouane et al., 2009),(Miladinovic et al., 2012) quant aux effets des essences d'autres lavandes envers $S$. aureus.

De façon pareille, les huiles essentielles de L. stoechas spp.stoechas récolée à l'ouest de la Turquie ont été rapportées être actives (de façon décroissante) sur $P$. mirabilis, Klebsiella pneumonia, Pseudomonas aeruginosa, E. coli, Staphylococcus aureus, Bacillus subtilis, et être sans activité sur Staphylococcus epidermidis et Enterococcus feacalis (Gören et al., 2002). De même, l'effet de $11 \mathrm{HE}$ de Lavandula stoechas sensu lato (L. virdis, L. stoechas; L. pedunculata, plus les sous-espèces et les hybrides) récoltée en Algérie a été positif sur des bactéries Gram+ «Bacillus subtilis », et des bactéries Gram négatives (E. coli, $K$. pneumoniae, Salmonella enterica; Pseudomonas aeruginosa (Benabdelkader et al., 2011). En fin, il a été rapporté que l'huile volatile de Lavandula stoechas L. en provenance de Tunisie a une activité antibactérienne envers six espèces bactériennes parmi lesquelles Staphylococcus aureus est la plus sensible (Bouzouita et al., 2005).

En revenant aux résultats du screening phytochimique et au dosage des polyphénols dans les extraits organiques qui ont révélé une différence qualitative et quantitative, on pourrait extrapoler cette différence également aux HE ; dès lors, le pouvoir antibactérien élevé chez Lavandula dentata en comparaison avec Lavandula pedunculata, pourrait être attribué à une différence de composition en principes actifs antimicrobiens.

De nombreux facteurs externes peuvent influencer la composition chimique de l'HE ; la température, le taux d'humidité, la durée d'ensoleillement, la composition du sol sont autant de facteurs d'ordre environnemental susceptibles d'induire des modifications qualitative et quantitative et engendrer ainsi un polymorphisme chimique dans une même espèce (Benabdelkader, 2012).

Une évaluation de la composition des HEs de populations algériennes de L. stoechas sauvages récoltées sur 11 sites différents dans le nord de l'Algérie a révélé un total de 121composés, avec seulement 66 substances 
communes à toutes les huiles, 1 à 3 composés majoritaires et un très grand nombre d'autres molécules, souvent présentes à l'état de trace (Benabdelkader, 2012). En Turquie, toujours chez la lavande stéchade, cette fois-ci au sens strict, 42 constituants ont été identifiés, dominés par le pulegone (Gören et al., 2002). En Tunisie, l'analyse de l'huile volatile de Lavandula stoechas L. , par CG et CG/SM a permis l'identification de 28 molécules dominées par la fenchone suivi par le camphre (Bouzouita et al., 2005). De plus, le rapport entre les éléments majoritaires peut varier dans des populations de lavande d'une zone géographique restreinte (Zrira et $a l$., 2003); et puis, selon (Ghalem et al., 2008) les composés majoritaires peuvent ne pas être nécessairement responsables de l'activité antibactérienne des HEs.

Quant au mode d'action des HEs sur les cellules bactériennes, il semble plus probable que l'activité antimicrobienne résulte de l'association conjointe de plusieurs mécanismes sur différentes cibles cellulaires (Burt, 2004). Ainsi, les composés phénoliques auraient pour cible la membrane plasmique et la paroi (Weber et al., 1996). D'autres agents compromettent plutôt l'intégrité structurale de la membrane plasmique en induisant une perte du matériel cytoplasmique (Reichling et al.,2002) au moment où la cible pour d'autres est probablement l'ADN bactérien suite à diffusion à travers les membranes bactériennes (Guessennd et al.,2008).

Outre les propriétés de l'HE, la sensibilité d'un microorganisme dépend du microorganisme lui-même (Kalemba et Kunicka, 2003) ; les bactéries Gram (+) sont plus sensibles aux HEs que les bactéries Gram (-), ceci revient en partie à la complexité de l'enveloppe cellulaire de ces dernières qui contient une double membrane, contrairement à la structure simple de la paroi des bactéries Gram (+). Plusieurs études testant l'activité inhibitrice des HEs confirment ce phénomène (Burt, 2004).

\section{Activité antifongique}

A la lumière des résultats reportés dans le tableau 3 , il apparait que les huiles des lavandes testées ont réduit de manière différente la croissance radiale des moisissures; de son côté, si le diamètre de la colonie fongique diminue avec l'augmentation de la concentration des huiles, cette diminution est surtout significative avec la concentration $0.5 \mu \mathrm{l} / \mathrm{ml}$.

Tableau 3 : Pourcentage d'inhibition (moyenne \pm écart type) de la croissance mycélienne Rhizopus stolonifer, de Penicillium expansum et Aspergillus brasiliensis en fonction de la concentration de l'huile essentielle.

$\begin{array}{ccccc}\text { Espèce } & \begin{array}{c}\text { Concentration } \\ \text { en } \mu 1 / \mathrm{ml}\end{array} & \text { A.brasiliensis } & \text { P.expansum } & \text { R.stolonifer } \\ & & \end{array}$




\begin{tabular}{cccrr}
\hline L.pedunculata & 0,125 & $14,81 \pm 1,70^{\mathrm{a}}$ & $3,81 \pm 3,60^{\mathrm{c}}$ & $0,00 \pm 0,00^{\mathrm{f}}$ \\
& 0,25 & $22,22 \pm 4,84^{\mathrm{a}}$ & $37,62 \pm 4,58^{\mathrm{d}}$ & $24,50 \pm 0,03^{\mathrm{g}}$ \\
& 0,5 & $40,74 \pm 1,70^{\mathrm{b}}$ & $75,24 \pm 3,20^{\mathrm{e}}$ & $93,33 \pm 0,00^{\mathrm{h}}$ \\
& & & & \\
\hline L.dentata & 0,125 & $11,85 \pm 0,64^{\mathrm{A}}$ & $18,57 \pm 3,78^{\mathrm{C}}$ & $0,00 \pm 0,00^{\mathrm{E}}$ \\
& 0,25 & $20,37 \pm 3,21^{\mathrm{A}}$ & $19,52 \pm 7,87^{\mathrm{C}}$ & $21,11 \pm 1,11^{\mathrm{F}}$ \\
& 0,5 & $37,04 \pm 3,39^{\mathrm{B}}$ & $63,33 \pm 8,61^{\mathrm{D}}$ & $80,37 \pm 0,64^{\mathrm{G}}$
\end{tabular}

*pour chaque espèce, Les moyennes sur la même colonne suivies de lettres différentes sont significativement différentes selon le test de Tukey au seuil de probabilité de $5 \%$.

Entre les trois champignons utilisés, quelle que soit la lavande considérée, Rhizopus stolonifer est le plus sensible et l'inhibition de sa croissance est presque totale à la concentration $0.5 \mu \mathrm{l} / \mathrm{ml}$, avec respectivement $93,33 \% \pm 0,00$ et $80,37 \% \pm 0,64$ pour $L$. pedunculata et $L$. dentata.

Aspergillus brasiliensis manifeste une certaine résistance en comparaison avec les autres moisissures même à des concentrations élevées des huiles testées $(0,5 \mu \mathrm{l} / \mathrm{ml})$; quant à la souche de Penicillium expansum, elle laisse voir une sensibilité proportionnelle aux concentrations des deux huiles, avec un effet d'inhibition de la lavande pédonculée généralement supérieur à celui causé par la lavande dentée.

Les résultats du test de l'activité antifongique révèlent qu'en plus de la différence de réaction aux HE entre les trois champignons, l'essence de la lavande pédonculée est plus active en comparaison à celle de lavande dentée. Ces résultats concordent avec ceux de Zuzarte et al., 2009 rapportant que l'huile essentielle de $L$.pedunculata a une importante activité antifongique. En effet, différents champignons impliqués dans des candidoses, dermatophytoses, aspergilloses ont été soumis aux effets des huiles essentielles de L. pedunculata récoltée au nord et au centre du Portugal ; un effet antifongique significatif fut observé vis-à-vis des dermatophytes d'une part, et d'autre part le chémotype riche en camphre fut le plus actif en comparaison avec celui à fenchone (Zuzarte et al., 2009).

Les HE de L. stoechas spp. Stoechas ont démontré une activité antifongique vis-à-vis de Rhizoctonia solani, Fusarium oxysporum alors que leur effet sur Aspergillus flavus était moindre (Angioni et al., 2006). Par ailleurs, $11 \mathrm{HE}$ de lavande stéchade algérienne furent actives sur des champignons filamenteux (Aspergillus niger, Fusarium oxysporum) et des levures (Candida albicans) reconnues pour leur impact clinique, sanitaire et économique (Benabdelkader et al., 2011).

Dans le même sens, les HE de Lavandula virdis testées sur des champignons filamenteux et des moisissures, ont eu un pouvoir antifongique 
sur différentes souches d'origine clinique (Candida albicans, C. krusei, C. guilliermondii), sur des dermatophytes cliniques (Epidermophyton floccosum FF9, Trichophyton mentagrophytes FF) ; ces mêmes HE furent peu effectives sur des Aspergillus (Aspergillus niger ATCC 16404 et A. fumigatus ATCC 46645 (Zuzarte et al., 2011).

L'activité antimicrobienne des HEs des plantes a été signalée comme étant liée spécifiquement à la présence de composés antimicrobiens. Récemment, des tentatives ont été faites pour identifier les composés responsables d'une telle bioactivité. Les molécules oxygénées qui entrent dans la composition des HEs sont généralement plus actives que les molécules hydrocarbonées qui sont, par contre, connues pour leurs faibles pouvoirs antimicrobiens (Kalemba et Kunicka, 2003).

$\mathrm{Vu}$ que les HE sont un mélange complexe de plusieurs composants, il est difficile d'attribuer leur activité biologique à un constituant particulier ; généralement, les composants majoritaires sont responsables du pouvoir antifongique (Bakkali et al., 2008). Toutefois, les composants minoritaires peuvent avoir un rôle crucial dans l'activité biologique des HEs (Koroch et al., 2007).

Le mécanisme d'action des HEs reste controversé ; certaines études avancent que ces composants peuvent pénétrer dans le micro-organisme et réagir avec les sites actifs d'enzymes et ou interférer avec le métabolisme cellulaire mais plusieurs propositions penchent vers une perturbation des membranes cellulaires et des effets pro-oxydants cytotoxiques (Bakkali. et al., 2008).

De façon générale, l'activité antimicrobienne est liée à la famille chimique des composés phénoliques dont la structure (noyau aromatique lié au groupement hydroxyle dans différentes positions), leur permet de former des liaisons hydrogènes avec les groupes-SH dans les sites actifs des enzymes cibles, ce qui entraîne la désactivation de ces enzymes dans les champignons (Ultee et $a l ., 2002$ ).

De même, les terpènes phénoliques agissent aussi en se fixant sur les groupes amines et hydroxylamines des protéines membranaires de la cellule microbienne en provoquant l'altération de la perméabilité et la fuite de contenus intracellulaires (Lopez-Malo et al., 2005).

Selon Bakkali et al. , 2008 dans le cas particulier de l'activité antifongique, l'action des $\mathrm{HE}$ inclurait une pénétration à travers les parois cellulaires et un endommagement direct du cytoplasme et des membranes mitochondriales vu l'arrêt rapide du métabolisme conduisant à la fin à la mort des cellules. 


\section{Conclusion}

Dans ce travail, les huiles essentielles des deux espèces de lavande investies se sont montrées actives contre les champignons et les bactéries testés ; alors que l'essence de L. pedunculta manifeste un effet inhibiteur très prononcé sur la croissance mycélienne, celle de L. dentata a plutôt un pouvoir antibactérien. Cette différence à lier fort probablement avec la composition chimique, soulignée par le screening chimique et le dosage des polyphénols devrait trouver justification via des analyses chromatographiques en phase gazeuse des huiles essentielles et un séquençage génétique des deux espèces de lavande.

Toujours est-il, à ce niveau d'étude, déjà, il faut insister sur la nécessité d'attirer l'attention quant à l'utilisation des lavandes uniquement sur la base du simple nom commun vu cette différence chimique qualitative et la différence de cibles sur lesquelles les deux espèces sont respectivement les plus actives.

\section{References:}

1. Angioni, A., Barra, A. et al.. Chemical composition, seasonal variability, and antifungal activity of Lavandula stoechas L. ssp. stoechas essential oils from stem/leaves and flowers. J. Agr. Food Chem. 54(12), 4364-4370,2006.

2. Bachiri.L., Labazi N., Daoudi, A.,Ibijbijen.J.,Nassiri.L.,Mokhtari, F., et Echchegadda G. Etude ethnobotanique de quelques lavandes marocaines spontanées Int. J. Biol. Chem. Sci., 9(3): 1308-1318, 2015.

3. Bakkali, F., Averbeck, S., Averbeck, D. \& Idaomar, M. Biological effects of essential oils - a review. Food Chem Toxicol 46, 446-475. Against dermatophyte, Aspergillus and Candida strains. J Appl Microbiol 100, 1333-1338, 2008.

4. Barchan, A.,Bakkali, M.,Arakrak, A.,Laglaoui ,A .Effet antibactérien et anti-biofilm de trois espèces de Mentha: Mentha spicata, Mentha pulegium et Mentha piperita, Phytothérapie; Lavoisier DOI 10.1007/s10298-015-0970-y ,2015.

5. Bekro,Y.A., Mamyrbekova, J.A., Boua,B.B., Ehile, E.E. Étude Ethnobotanique et Screening phytochimique de Caesalpinia benthamiana (Baill.) Herend. rt Zarucchi (Caesalpiniaceae). Sciences \& Nature Vol. 4, No. 2, 217-225, 2007.

6. Bellakhdar J. Médecine arabe ancienne et savoir populaire. La pharmacopée traditionnelle Marocaine, Ibis Press, Paris. p : 759, 1997

7. Benabdelkader,T., Zitouni, A., Guitton, Y., Jullien, F., Maitre, D., Casabianca, H., Legendre,L., and Kameli,A. Essential oils from wild 
populations of algerian Lavandula stoechas L.: composition, chemical variability and in vitro biological properties. Chemistry \& Biodiversity 8(5), 937-953 ,2011.

8. Benabdelkader., T. Biodiversité, bioactivité et biosynthèse des composés terpéniques volatils des lavandes ailées, Lavandula stoechas sensu lato, un complexe d'espèces méditerranéennes d'intérêt pharmacologique. Biologie végétale. Université Jean Monnet - Saint-Etienne ; Ecole normale supérieure de Kouba (Alger), 2012.

9. Bouzouita, N., Kachouri, F. et al. Volatile constituents and antimicrobial activity of Lavandula stoechas L. oil from Tunisia. J. Essent. Oil Res. 17, 584-586,2005.

10. Bruneton, J.Pharmacognosie, Phytochimie, Plantes médicinales, 2éme édition, Technique et Documentation, Lavoisier, Paris,915,1993.

11. Burt, S., Essential oils: their antibacterial properties and potential applications in foods-a review. International Journal of Food Microbiology, 94, 223-253, 2004.

12. Cavanagh, H., M. A. \& Wilkinson, J. M. Biological activities of lavender essential oil. Phytother Res16, 301-308, 2002.

13. Chambers H. F., 1997.Methicillin resistance in staphylococci: molecular and biochemical basis and clinical implications. Clin. Microbiol. Rev. 10: 781-791.

14. Clevenger, J.F., 1928. Apparatus for the Determination of Volatile Oil. Journal of the American Pharmaceutical Association, Vol. 17, No. 4,345-349, 1928.

15. Dadalioglu, I., \& Evrendilek, G.A.Chemical compositions and antibacterial effects of essential oils of Turkish oregano (Origanum minutiflorum), bay laurel (Laurus nobilis), Spanish lavender (Lavandula stoechas L.), and fennel (Foeniculum vulgare) on common foodborne pathogens. J Agric Food Chem 52, 8255-8260, 2004

16. Dohou,N. Screening phytochimique d'une endémique ibéromarocaine thymelaea lythroides, Bull. Soc. Pharm. Bordeaux 142, 61-78, 2003.

17. Falleh, H., Ksouri, R., Chaieb, K., Karray-Bouraoui, N., Trabelsi, N., Boulaaba, M., et Abdelly, C. Comptes Rendus Biologies, 331 :372379,2008

18. Ghalem, B. and Mohamed, B. Antibacterial activity of leaf oils of Eucalyptus globules and Eucalyptus camaldulensis; Afr. J. Pharmacol.2, 211-215, 2008. 
19. Gören, A. C., Topçu, G., et al. The chemical constituents and biological activity of essential oil of Lavandula stoechas ssp. stoechas. Z. Naturforsch 57c, 797-800,2002.

20. Guessennd, N., Kacou-N'douba, A., et al. Prévalence et profil de résistance des entérobactéries productrices de -lactamase à spectre élargie (BLSE) à Abidjan Côte d'Ivoire de 2005 à 2006. J. Sci. Pharm. Biol. 9(1), 63-70, 2008.

21. Hyldgaard, M., Mygind, T., and Meyer, R.L. Essential oils in food preservation: mode of action, synergies, and interactions with food matrix components. Frontiers in Microbiology,3, 1-24,2012.

22. Imelouane, B., Elbachiri,A., AnkiT, M., Benzeid,H., and Khedid, K. Physico-Chemical Compositions and Antimicrobial Activity of Essential Oil of Eastern Moroccan Lavandula dentata, International Journal of Agriculture \& Biology, ISSN:1560-8530, 113-118, 2009

23. Kalemba, D., and Kunicka A. Antibacterial and antifungal properties of essential oils. Curr. Med. Chem: 10,813-829, 2003

24. Koroch, A., Juliani, R., H. \& Zygadlo, J. A. Bioactivity of essential oils and their components. In Flavours and Fragrances. Chemistry, Bioprocessing and Sustainability, Edited by R. G. Berger. Berlin, Heidelberg: Springer-Verlag. 87-115, 2007.

25. Lang, G., and Buchbauer, G. A review on recent research results (2008-2010) on essential oils asantimicrobials and antifungals. A review. Flavour and Flagrance Journal, 27, 13-39, 2011.

26. Le Loir,Y et Gautier,M. .Monographies de microbiologie : Satphlococcus aureus ,Edition Lavoisier,233 2010

27. Lopez-Malo, A., Alzamora, SM., Palou, E. Aspergillus flavus growth in the presence of chemical preservatives and naturally occurring antimicrobial compounds. Int J Food Microbiol 99:119-128, 2005.

28. Matos, F., Miguel, M. G., Duarte, J., Venancio, F., Moiteiro, C., Correia, A. I. D., Figueiredo, A. C., Barroso, J. G. \& Pedro, L. G. Antioxidant capacity of the essential oils from Lavandula luisieri, $L$. stoechas subsp. lusitanica, L. stoechas subsp. lusitanica x L. luisieri and L. viridis grown in Algarve (Portugal). JEOR, 21, 327-336, 2009.

29. Miladinovic, D., Ilic, B., Mihajilov-Krestv, T., Nikolic, N., Miladinovicl,. \& Cvetkovic, O., Investigation of the chemical composition antibacterial activity relationship of essential oils by chemometric methods. Analytical and bioanalytical chemistry, 403:1007-18, 2012.

30. Zuzarte, M., Jose Goncalves, M., Cavaleiro,C., Canhoto, J., ValeSilva, L., Joao Silva, M., Pinto,E., and Ligia Salgueiro. Chemical 
composition and antifungal activity of the essential oils of Lavandula viridis. Journal of Medical Microbiology, 60, 612-618, 2011.

31. Moon, T., Wilkinson, J. M. \& Cavanagh, H. M. A. Antibacterial activity of essential oils, hydrosols and plant extracts from Australian grown Lavandula spp. Int J Aromatherapy. 16, 9-14. 2006

32. Pandey, D.K., Tripathi, N.N., Tripathi, R.D., Dixit, S.N, Fungitoxic and phytotoxic properties of essential oils of Hyptis suaveolens. Z. Pflanzenkranjheiten und Pflanzenschutz 89: 344-349 J. Plant. Dis. Prot.89; 344-349, 1982.

33. Perrucci, S., Macchioni, G., Cioni, P. C., Flamini, G., Morelli, I. \& Taccini, F. The activity of volatile compounds from Lavandula angustifolia against Psoroptes cuniculi. Phytother Res, 10, 5-8, 1996

34. Reichling, J., Weseler, A., et al. Bioactive essential oils used in phytomedicine as anti-infective agents: Australian tea tree oil and manuka oil. Act. Pythoter, 1, 26-32. 2002

35. Rhayour, K. Etude du mécanisme de l'action bactéricide des huiles essentielles sur Esherichia coli, Bacillus subtilis et sur Mycobacterium phlei et Mycobacterium fortuitum. Thèse de doctorat. Université Sidi Mohamed Ben Abdellah. Fès, Maroc, 170.2002

36. Smallfield.B., Introduction to growing herbs for essential oils, medicinal and culinary purposes. Crop and Food Research 45:1-4, 2001.

37. Soylu, E.M., Kurt, S., Soylu, S. In vitro and in vivo antifungal activities of the essential oils of various plants against tomato grey mould disease agent Botrytis cinerea, Int. J. Food. Microbiol. 143:183-189, 2010.

38. Svoboda, K.P., Hampson, J.B., Bioactivity of essential oils of selected temperate aromatic plants: antibacterial, antioxidant, antiinflammatory and other related pharmacological activities. Ed: Plant Biology Department, 1999.

39. Ultee, A., Bennink, M.H.J. and Moezelaar, R. The phenolic hydroxyl group of carvacrol is essential for action against the food-borne pathogen Bacillus cereus. Applied and Environmental Microbiology ().4, 1561-1568, 2002.

40. Vermerris, W., Nicholson, R. Isolation and Identification of Phenolic Compounds, Phenolic Compound Biochemistry. Dordrecht: Springer, 1-70, 2006.

41. Weber, F.J., and de Bont, J. A. M. Adaptation mechanisms of microorganisms to the toxic effects of organic solvents on membranes. Biochimi. Biophys. Acta.12, 225245, 1996. 
42. Zrira, S., and Benjilali, B.The constituents of the oils of Lavandula stoechas L. ssp. atlantica Br.-Bl. and L. stoechas ssp. stoechas from Morocco. J. Essent. OilRes. 15, 68-69. 2003.

43. Zuzarte, M., Gonçalves, M. J. et al.. Chemical composition and antifungal activity of the essential oils of Lavandula pedunculata (Miller) Cav. Chem. Biodivers 6, 1283-1292, 2009. 